Argumentation et Analyse du Discours

\title{
Praxis et anti-praxis des revues intellectuelles de l'immédiat après-guerre
}

Praxis and anti-praxis in the intellectual journals of the immediate afterwar

\section{Thomas Franck}

\section{(2) OpenEdition \\ 1 Journals}

Édition électronique

URL : http://journals.openedition.org/aad/2470

DOI : 10.4000/aad.2470

ISSN : $1565-8961$

Éditeur

Université de Tel-Aviv

Référence électronique

Thomas Franck, "Praxis et anti-praxis des revues intellectuelles de l'immédiat après-guerre », Argumentation et Analyse du Discours [En ligne], 19 | 2017, mis en ligne le 15 octobre 2017, consulté le 23 septembre 2019. URL : http://journals.openedition.org/aad/2470; DOI : 10.4000/aad.2470

Ce document a été généré automatiquement le 23 septembre 2019.

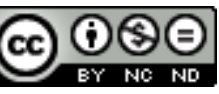

Argumentation \& analyse du discours est mis à disposition selon les termes de la licence Creative Commons Attribution - Pas d'Utilisation Commerciale - Pas de Modification 4.0 International. 


\title{
Praxis et anti-praxis des revues intellectuelles de l'immédiat après- guerre
}

\author{
Praxis and anti-praxis in the intellectual journals of the immediate afterwar
}

\author{
Thomas Franck
}

\section{Dialogique discursive et idéologie dialectique}

1 Les textes de présentation des revues intellectuelles créées après 1945 permettent d'interroger la dimension collective de discours dont les intentions sont non seulement de se positionner et de se définir positivement, mais aussi de réfuter une conception du travail intellectuel à laquelle elles n'adhèrent pas. Le cas particulier de la revue Socialisme ou Barbarie occupe ici une place déterminante en raison de sa dimension ouvertement collective, critique et militante. Il est néanmoins nécessaire de la replacer dans un espace plus large afin de comprendre les logiques de positions et d'oppositions idéologiques qui parcourent ces revues intellectuelles. Dans ce cadre, les textes de présentation de Critique, Les Temps Modernes, La Jeune Révolution, La Table Ronde et La Nouvelle Critique constituent un matériau qui permet de dégager plusieurs tendances rhétoriques propres au discours revuistique de l'immédiat après-guerre. À l'aide d'une approche située à la croisée de l'analyse du discours contemporaine et de la philosophie politique sartrienne, nous tentons de montrer la manière dont ces productions, conçues au travers de leurs matérialités historique, discursive et éditoriale, favorisent l'émergence de débats plus ou moins polémiques et oppositionnels autour du marxisme et des questionnements induits par cette idéologie sur la dimension collective de l'action intellectuelle.

2 L'attention portée au marxisme se justifie par la prolifération des discours le concernant dans les revues de l'immédiat après-guerre et par le besoin réel qu'éprouvent les intellectuels français de se positionner collectivement par rapport au P.C.F., aux politiques et à l'idéologie de l'URSS ainsi qu'aux autres mouvances marxistes 
ou d'extrême gauche - un constat notamment réalisé par Paul Veyne (2014: 75), Frédérique Matonti (2004), Anna Boschetti (1985: 195-196) ainsi que par Pascal Ory et Jean-François Sirinelli (2002: 150-154). Ces derniers fournissent un commentaire fondamental, évoquant la "légitimité idéologique », voire "l'hégémonie intellectuelle ", dont est doté le communisme dans les années d'immédiat aprèsguerre :

L'influence du communisme dépasse alors, indirectement, cette large mouvance composée d'un noyau de clercs "encartés» et d'un conglomérat de «compagnons». Un véritable "philocommunisme» s'est emparé de nombre d'autres clercs, qui campent aux franges de cette mouvance, sans franchir le fossé. [...] En d'autres termes, le Parti communiste, même sur ses franges, offre à l'intellectuel une action concrète et apparemment efficace, satisfait son souci de cohérence et nourrit son espérance (ibid. : 153).

3 Ainsi, les contextes discursifs et historiques de la création et du développement des revues étudiées (d'octobre 1945 à avril 1949) nous obligent à tenir compte, en plus de leurs positionnements idéologiques, de la situation politique de l'immédiat aprèsguerre. Cette situation influence en effet l'autodéfinition des revues ${ }^{1}$, dont les présentations sont explicitement ou implicitement marquées par les querelles idéologiques et politiques. L'importance qu'accorde le monde intellectuel français à l'idéologie marxiste entraîne, au sein des revues, une série de questionnements relatifs aux consignes théoriques et pratiques d'un organe collectif ; elle mène à s'interroger sur l'engagement de l'intellectuel, le rôle critique de la revue et ses positions par rapport aux logiques de parti - autant d'éléments déterminés par un rapport à l'action de l'intellectuel telle que la pensent les diverses obédiences marxistes.

Les textes de présentation des revues intellectuelles constituent dès lors une réelle position au sein de la formation discursive marxiste (déterminant les discours relatifs à l'action critique de l'intellectuel au sein d'un groupe), au sens où l'entend Michel Pêcheux. Celui-ci caractérise la formation discursive comme un ensemble énonciatif relativement cohérent établissant ce qui peut et doit être dit à un moment et dans une situation donnés, comme un espace de régularités déterminé par des structures discursives propres (genre, récurrences rhétoriques, topoï communs, fonctionnement éditorial, etc.) et par une idéologie agencée suivant diverses positions et oppositions (voir à ce propos Pêcheux 1975, Pêcheux 1990, Maingueneau 2011 et Angermuller 2013b). Le caractère plus ou moins affirmatif et polémique des textes de présentation, parfois à la limite du pamphlétaire, induit une série de particularités rhétoricoargumentatives significatives (unités topiques) traduisant des positions idéologiques au sein du monde intellectuel (voir Maingueneau 2011). Les textes de présentation de revues telles que Socialisme ou Barbarie, en interrogeant la dimension collective et critique de leur action, peuvent dès lors être considérés comme des positionnements déterminants de la formation discursive marxiste. Évoquons ici quelques-uns des traits dégagés, dans le cadre de nos travaux récents, dans les textes de présentation des Temps Modernes, de Critique et de La Table Ronde et qui seront mis en regard de la singularité discursive de l'éditorial de Socialisme ou Barbarie: attaques ad hominem, questions rhétoriques, allo-attributions par îlots textuels, registre polémique, vocabulaire militaire de l'attaque-défense, ironie envers l'adversaire, ethos pamphlétaire du prophète contre ethos de modestie, topos de la "libre discussion " contre topos des partis pris idéologiques limitant le débat. 
5 En raison des singularités propres au format éditorial des revues (fragmentation, contraintes de place, exigences rédactionnelles et typographiques) et de la temporalité que celles-ci privilégient (périodicité, rapidité et succession des publications), un rapport particulier se crée entre les savoirs véhiculés et la matérialité qui les définit. En effet, en transmettant un état momentané d'une réflexion intellectuelle (ses dynamiques, ses dialogues et ses déplacements), le format de la revue, et plus encore celui du texte de présentation, permettent de saisir sur le vif un savoir collectif en cours d'élaboration et en dialogue constant. En outre, l'urgence des situations historiques dans lesquelles s'insèrent ces discours collectifs d'après-guerre participe à une mise en lumière de leur dimension idéologique. Cette urgence constitue dans le même temps la revue comme une «mémoire immédiate » (Curatolo et Poirier 2002:3) faisant état d'une histoire des croyances collectives. Le rythme des publications et de la création des revues, les stratégies de positionnement idéologique de leurs manifestes, la circulation de différents concepts, les trajectoires des intellectuels au sein des revues ainsi que la nécessité de répondre aux besoins d'un lectorat rendent ces discours intrinsèquement dynamiques, dialogiques et critiques, suivant une constante interaction entre les structures sociales, les idées qui en émergent et les acteurs en lutte. Dans Le Champ de la théorie, Johannes Angermuller a mis en avant le rôle des revues intellectuelles, des Temps Modernes aux Actes de la recherche en sciences sociales, dans les «transformations profondes dans l'"économie politique" du discours intellectuel » (Angermuller 2013a: 80). Il sera ici question d'analyser un cas singulier et significatif d'une revue influençant le discours intellectuel en reprenant le projet d'Angermuller d'une étude du champ théorique, projet que nous entendons déplacer vers une dimension sociodiscursive.

6 Tous ces éléments relatifs à la matérialité (éditoriale, discursive et sociohistorique) de la revue intellectuelle amènent à considérer celle-ci comme un discours d'intervention, c'est-à-dire comme une production agissant sur les structures sociales qui lui sont propres. Cette notion d'intervention doit être comprise suivant deux acceptions, d'une part celle d'une action transformatrice déplaçant l'institution d'une formation discursive et d'autre part, suivant l'étymologie de l'infinitif inter-venire, celle d'un positionnement à l'intersection de différentes productions déjà instituées, au cœur du déjà-dit, du déjà-pensé, à la croisée des positions propres à une formation discursive² Comme le note Yves Peyré, on trouve, d'une part, un « acte militant à la racine même de la revue » (2002: 8) et, d'autre part, la réponse au besoin de mettre en scène, de rejouer, par une polyphonie de voix, un dialogue agissant sur la réalité sociale et se construisant au travers de débats, de dissonances et de dissensions. Il sera donc question de montrer en quoi la revue intellectuelle d'après-guerre est foncièrement déterminée par un projet d'intervention sociale et par une visée critique que traduisent les structures rhétoriques et argumentatives des textes de présentation. Pour ce faire, nous mobilisons les notions sartriennes de praxis et d'anti-praxis (voir, principalement, Sartre 1960 et 2015) qui sous-tendent l'ensemble de cette recherche et qui permettent de comprendre le projet dialectique de la revue, conçue à la fois comme sujet collectif instituant et comme matérialité discursive instituée et déterminée par les structures rhétoriques d'une formation discursive.

7 En étendant les réflexions sartriennes relatives au pouvoir de la matière sur l'action humaine au matériau discursif, nous tenterons de comprendre la manière dont interagissent un discours de groupe singulier et la formation discursive dans laquelle il 
s'insère: "la matière comme limite de la signification se fait la médiation des significations entre elles. C'est en elle et par elle que les significations (praxis cristallisée) se combinent en une synthèse neuve mais toujours inerte » (Sartre 1960 : 278). Ainsi, si l'innovation rhétorique peut être pensée comme une praxis humaine agissant sur la matière discursive, celle-ci peut également contraindre, par son inertie, l'innovation telle une anti-praxis agissant sur les groupes humains en tant que " praxis sans auteur» (ibid.: 276). Nous tenterons de comprendre et de dépasser cette dialectique, tout en en évaluant les implications dans le cadre d'une analyse sociodiscursive, grâce à l'étude du cas particulier des textes de présentation des revues intellectuelles de l'immédiat après-guerre.

\section{Hétérodoxie antistalinienne et dogmatisme de groupe}

8 La présentation de Socialisme ou Barbarie s'ouvre sur un positionnement idéologique explicite qui rompt tant avec la «bureaucratie stalinienne» qu'avec l'« attitude réformiste » de la «IV internationale trotskiste » (Socialisme ou Barbarie 1949:1) dont elle est pourtant l'un des produits. Cette posture explicite d'autodétermination groupale, traduite notamment par le syntagme introductif « Le groupe dont cette revue est l'organe », présuppose l'existence du groupe comme un a priori. Si cette affirmation collective de soi peut apparaître comme évidente dans le cadre de la définition d'une revue intellectuelle militante, elle est en réalité le fruit d'une évolution des pratiques discursives et des conflits politiques qui les influencent. En effet Les Temps Modernes, en octobre 1945, puis Critique, en juin 1946, ne se risquent nullement à un positionnement aussi clair et univoque ni à une affirmation aussi évidente de la cohésion de leur groupe, mais questionnent au contraire leur unité problématique. Bien que leur projet ne corresponde pas au programme militant de Socialisme ou Barbarie, il est toutefois utile de noter une évolution discursive représentative de l'instauration progressive d'un contexte politique de guerre froide incitant les organes collectifs à l'explicitation de leurs positions idéologiques, contrairement à la prudence des revues créées immédiatement après la guerre: le texte de présentation des Temps Modernes reste beaucoup plus prudent et mesuré - « Nous abordons tous l'étude de ces problèmes dans un esprit commun; mais nous n'avons pas de programme politique; chaque article n'engagera que son auteur " (Sartre 1945: 19) - tout comme celui de Critique - «Les auteurs des articles développent librement une opinion qui n'engage qu'eux-mêmes, ils cherchent à fonder cette opinion en raison sans se contenter des facilités polémiques " (Critique 1946: 2). Beaucoup plus assertive et péremptoire est la formulation par Socialisme ou Barbarie de sa "conception d'ensemble », "systématique et cohérente »: "Cette revue n'est nullement un organe de confrontation d'opinions entre gens qui "se posent des problèmes", mais l'instrument d'expression d'une conception d'ensemble que nous croyons systématique et cohérente » (Socialisme ou Barbarie : 6).

9 Ce déplacement politique d'une logique collective sérielle à une pratique de groupe organisé (voir Sartre 1960: 449-511) et cette accentuation rhétorique du caractère assertif et unifié de la revue se voient déterminés par trois facteurs : (1) une évolution de l'espace des revues intellectuelles qui réoriente les débats relatifs au marxisme, notamment en réaction au dogmatisme de La Nouvelle Critique et à la conception bourgeoise de l'engagement intellectuel des Temps Modernes, (2) un ensemble de 
facteurs socio-historiques et politiques, dont l'instauration d'un contexte de guerre froide, et (3) un projet inhérent à la revue elle-même et à son programme idéologique, qui assume ouvertement une visée militante non orthodoxe. Nous nous arrêtons dans un premier temps sur l'évolution de l'espace des revues intellectuelles ayant influencé le développement de cette rhétorique de l'affirmation assertive d'un groupe et de son idéologie, pour ensuite étudier l'influence du contexte historico-politique sur cette rhétorique et enfin analyser le projet militant de Socialisme ou Barbarie conçu comme praxis de groupe.

10 Si plusieurs revues d'obédience communiste existaient déjà en France avant 1945, telles que La Pensée, La Vérité, Les Lettres Françaises ou encore La Révolution prolétarienne, une revue fondamentale est créée en décembre 1948, revue par rapport à laquelle Socialisme ou Barbarie se positionne directement, à savoir La Nouvelle Critique. Par ailleurs, à la suite de l'interdiction de la N.R.F. pour collaboration (Paulhan intègre le comité de rédaction des Temps Modernes et s'occupe d'une revue essentiellement littéraire, Les Cahiers de la Pléiade), Les Temps Modernes et Critique structurent dans un premier temps, avec Esprit (créée en 1932), l'horizon des revues intellectuelles de 1946 à 1948. Vient s'ajouter à cette tripartition la revue $L a$ Table Ronde, créée en janvier 1948 par Mauriac, qui entend quant à elle déplacer, en s'y confrontant explicitement, l'engagement existentialiste des Temps Modernes en privilégiant une «liberté de l'esprit» débarrassée de toutes « consignes que dicte un parti » (La Table Ronde $1948: 4$ ).

11 Les relations difficiles de Sartre et des Temps Modernes avec le P.C.F., l'hostilité des acteurs d'Esprit et de La Table Ronde envers le communisme ainsi que l'hétérodoxie savante de Critique laissent en quelque sorte ouverte la place aux positions communistes orthodoxes qui trouvent dans La Nouvelle Critique, créée par Jean Kanapa sous l'autorité du P.C.F., un lieu d'expression d'une ferveur militante, orthodoxe et dogmatique :

C'est cette «bataille idéologique incessante » que La NOUVELLE CRITIQUE entend mener. Ce sont ces deux tâches indispensablement liées de critique de l'adversaire réactionnaire et de diffusion de la pensée marxiste-léniniste-stalinienne qu'elle entend remplir (La Nouvelle Critique 1948 : 3-5).

Boschetti voit dans cette revue à la fois une volonté de concurrencer Les Temps Modernes et son "ambition totalisante", ambition propre à l'anthropologie phénoménologique sartrienne mais convenant au projet marxiste, et en même temps un " envers négatif ", un travail du prolétariat éclairé ou d'une avant-garde parlant en son nom contre un projet prétendument issu de la bourgeoisie, classe amalgamée dans le discours communiste à l'ensemble des intellectuels (ibid. : 14). Dans la continuité de ce constat, la sociologue réalise un commentaire permettant de comprendre la position de Socialisme ou Barbarie par rapport à celle de La Nouvelle Critique et à celle des Temps Modernes: La Nouvelle Critique se pose «en rivale des revues intellectuelles "bourgeoises". Mais ce défi apparaît dérisoire dès que l'on compare leur capital à celui des existentialistes » (Boschetti 1985 : 216).

13 Face à cette absence de réel capital symbolique et culturel (si on la compare aux autres revues intellectuelles animées par des acteurs majeurs de la pensée française de l'immédiat après-guerre) et face au caractère prescriptif, jugeant, normatif et dogmatique des analyses de La Nouvelle Critique, Socialisme ou Barbarie vient dans une certaine mesure pallier, avec des intellectuels tels que Claude Lefort et Cornelius Castoriadis $^{3}$ (la tendance Chaulieu-Montal ${ }^{4}$ ) ou Jean Laplanche ${ }^{5}$ (écrivant sous le 
pseudonyme de Marc Foucault), ce manque de légitimité et de rigueur intellectuelle, tout en prétendant s'adresser à l'« avant-garde des ouvriers manuels et intellectuels " (Socialisme ou Barbarie 1949 : 2). C'est ce que suggère Philippe Gottraux, dans sa thèse sur Socialisme ou Barbarie, dans une mise en perspective de l'hégémonie intellectuelle et symbolique des Temps Modernes, de la «hargne communiste " de La Nouvelle Critique et du projet intellectuel-militant de Socialisme ou Barbarie (1997: 255-314). Contre la dimension bourgeoise et individualisante des Temps Modernes et contre la violence dogmatique et autoritaire des intellectuels peu légitimés de La Nouvelle Critique, la revue Socialisme ou Barbarie ouvre une troisième voie, intellectualiste mais prolétarienne, qui laisse place à une position discursive, celle du marxisme hétérodoxe et antistalinien dont nous allons analyser les particularités rhétorico-argumentatives. L'impact de la revue de Lefort et Castoriadis est cependant véritablement limité, sa légitimité ne se réalisant que tardivement et à rebours. Toutefois, il apparaît essentiel de mettre en lumière les singularités rhétorico-idéologiques de cette revue, certes minoritaire dans le monde intellectuel et revuistique de la fin des années 1940, qui traduit et prépare, discursivement et idéologiquement, une conception du travail intellectuel qui influencera plusieurs organes collectifs des années 1950, que l'on pense sommairement à Preuves (1951), Arguments (1956) ou Partisans (1961).

\subsection{Un registre polémique : entre libre discussion et parti pris idéologique}

Dans un premier temps, on peut aisément voir dans le commentaire ci-dessous du texte de présentation de Socialisme ou Barbarie une adresse, du moins une allusion, aux revues marxistes orthodoxes - et principalement à La Nouvelle Critique, dite «du marxisme militant » :

Nous pensons que nous représentons la continuation vivante du marxisme dans le cadre de la société contemporaine. Dans ce sens nous n'avons nullement peur d'être confondus avec tous les éditeurs de revues "marxistes", "clarificateurs", "hommes de bonne volonté », discutailleurs et bavards de tout acabit. Si nous posons des problèmes, c'est que nous pensons pouvoir les résoudre (Socialisme ou Barbarie 1949 : 3).

Véritable rhétorique propre aux débats revuistiques, les attaques ad hominem qui ne sont pas placées entre guillemets mais assumées par le locuteur - «discutailleurs et bavards de tout acabit " - de même que l'humour de l'évidence faisant allusion à la naïveté de l'adversaire - «Si nous posons des problèmes, c'est que nous pensons pouvoir les résoudre" - ainsi que l'utilisation des guillemets permettant une distanciation par usage ironique de la citation (que l'on nommera dans ce cas «îlot textuel ironique $\left.»^{6}\right)$, situent ce discours dans une formation discursive polémique. Au sein de celle-ci se construit une apparence de dialogue ; derrière elle se profilent des antagonismes idéologiques et un dialogue de sourds. Conjointement à lîlot textuel ironique des revues dites " marxistes", on peut voir dans celui des " hommes de bonne volonté » une moquerie de l'obsession discursive de l'honnêteté et de la sincérité des communistes staliniens: en effet, La Nouvelle Critique use du terme "honnête» de manière véritablement obsessionnelle (parfois plus de cinq occurrences par page dans son texte de présentation), comme un besoin d'autojustification face aux " mensonges " des intellectuels bourgeois, qualifiés de "trifouilleurs de poubelles" (La Nouvelle Critique 1948 : 14). 
16 À l'appui de cette mise en lumière de la dimension polémique des textes de présentation des revues, il est intéressant de noter que la plupart d'entre eux s'ouvrent sur une attaque envers l'adversaire et sur un positionnement par négation, par réfutation antithétique. C'est le cas des Temps Modernes qui dénonce l'écrivain bourgeois réaliste, de La Nouvelle Critique qui attaque à la fois le gouvernement français, les écrivains collaborateurs, les productions pornographiques américaines et les fascistes, ou encore de La Table Ronde qui consacre l'entame de son " Au lecteur » à dire ce qu'elle n'est pas - «Il y a beaucoup de revues, et de toutes sortes. [...]. [La Table Ronde] n'est pas un recueil d'anthologie de textes littéraires. Elle n'est pas davantage un organe périodique d'information commentée, ni la chaire d'enseignement d'une école, ni l'instrument de propagande intellectuelle d'un parti politique » (La Table Ronde 1948 : 3). Socialisme ou Barbarie ne déroge pas à cette règle polémique selon laquelle on se définit avant tout par ce qu'on n'est pas.

Les termes «bavards de tout acabit » de l'éditorial de Socialisme ou Barbarie peuvent en outre faire écho à l'allusion de Lefort, dans un article pour La Jeune Révolution, revue des étudiants du PCI, aux «bavardages philosophiques » (Lefort 1946a: 8) des intellectuels communistes. De même, l'allusion humoristique «Si nous posons des problèmes, c'est que nous pensons pouvoir les résoudre » fonctionne de manière analogue (c'est-à-dire par tournure hypothétique et par reformulation moqueuse d'une évidence) à la critique acerbe que formule Lefort à propos de Naville, militant communiste à l'origine de $L a$ Revue internationale, dans un article paru dans Les Temps Modernes en 1946: «[...] personne n'obligeait Naville à écrire des Essais philosophiques [...]. Mais s'il veut faire de la philosophie, qu'il ne prenne pas le ton du savant, qu'il ne vienne pas avec sa véracité préétablie du genre : deux et deux font quatre, ou bien : il suffit de se heurter à une chaise pour savoir que le monde existe" (Lefort 1946b:142). Les similitudes rhétoriques sont assez évidentes entre ces différentes productions et participent, par leur usage de l'humour et de l'ironie, au renforcement d'une logique polémique propre au discours intellectuel marxiste d'après-guerre.

18 Contre le dogmatisme stalinien de La Nouvelle Critique, contre l'orthodoxie trotskiste de La Jeune Révolution (revue avec laquelle le groupe avait dans un premier temps collaboré) et contre l'idéal d'une libre discussion débarrassée de tout a priori idéologique, tel que l'explicitent Les Temps Modernes mais surtout Critique et La Table Ronde, la revue Socialisme ou Barbarie développe un commentaire réflexif ayant conscience des positions idéologico-discursives à l'origine de tout dialogue intellectuel : «La discussion sera donc libre dans le cadre de nos conceptions générales, avec le souci constant d'éviter que cette discussion ne devienne un dialogue sans fin entre quelques individus » (Socialisme ou Barbarie 1949:6, nous soulignons). Cette question du rapport entre d'une part les cadres idéologico-discursifs antagonistes participant à la constitution d'un registre de dispute et d'autre part la libre discussion totale et sans entrave constitue un véritable topos d'époque. Elle est une obsession discursive, en ce sens qu'elle induit un mécanisme argumentatif dialectique plus ou moins stéréotypé et présenté comme inévitable et essentiel (la thèse du libre examen est opposée à l'antithèse du parti pris idéologique donnant lieu à une synthèse pouvant être résumée par "polémique mais ouvert au dialogue »). Les textes de présentation des revues reviennent presque tous sur ce topos, de même que de nombreux articles relatifs aux oppositions idéologiques induites par le commentaire marxiste. 
19 Nous citerons quatre articles, parus de 1946 à 1948, démontrant que les intellectuels et les revues considérés comme libéraux sont également pris dans les logiques de luttes idéologiques dont traite l'analyse marxiste. On le voit premièrement dans l'article de Roland Caillois « La pensée politique de Raymond Aron » paru dans Critique - « la liberté n'est plus réclamée par les uns et les autres mais elle est l'enjeu de la lutte, la "libre" discussion n'est plus qu'un aspect de la lutte à mort, on ne la réclame qu'à des moments opportuns et pour des raisons précises » (Caillois 1946: 436) ; ensuite dans «Marxisme ou cartésianisme » de Ferdinand Alquié paru dans Les Temps Modernes - pour qui « notre temps est moins celui des discussions que celui des querelles » (Alquié 1946:1378) - ou encore dans « À propos du matérialisme dialectique » d'Éric Weil publié dans Critique considérant évoluer dans une « époque où il est difficile de s'entendre [...]. Rien de plus naturel dans un tel moment que de prendre position, ouvertement, avec violence même. Cependant, il y a une condition : il ne faut pas fausser les issues " (Weil 1946 : 83). Enfin, c'est la présentation de La Table Ronde qui réalise la synthèse de ce topos dialectique de la « libre discussion contrainte », exemplairement structuré suivant une argumentation en trois moments («Ce qui est certain [...] C'est au nom de [...] Ce faisant ») :

(1) Ce qui est certain, c'est que dans les temps où s'affrontent les fanatismes, la liberté de l'esprit constitue une forme d'engagement aussi honorable que l'adhésion passionnée ou prudente à une faction militante : aussi honorable, et au moins aussi périlleuse.

(2) C'est au nom de la liberté de l'esprit qu'il arrivera aux collaborateurs de cette revue de prendre position: soit pour dénoncer des impostures; soit pour tenter de rendre plus clairs des problèmes mal posés, car nous débattons au milieu des impostures, au milieu des problèmes mal posés.

(3) Ce faisant, ceux dont le lecteur verra la signature, dans les pages qui suivent, estiment qu'ils feront, tout simplement, leur métier : car leur métier est, autant que possible, de voir clair; et leur métier est de prendre la parole, même et surtout lorsque tout le monde garde le silence - leur métier est de rompre le silence (La Table Ronde $1948: 4$; nous soulignons les structures argumentatives organisant de manière dialectique le topos de la libre discussion contrainte).

On peut dès lors analyser, d'un point de vue sociodiscursif, la position de Socialisme ou Barbarie dans son étroite relation avec les textes de présentation des autres revues qui actualisent les structures rhétorico-argumentatives d'une formation discursive instituée (topos de la liberté d'esprit régie par des cadres idéologiques, attaques ad hominem et îlots textuels ironiques, contraintes de l'engagement de l'intellectuel, position par réfutation antithétique, rapport à l'action collective) tout en développant un nouveau discours instituant qui déplace cette formation (ouverture à un discours marxiste hétérodoxe antistalinien, ni trotskiste, ni anarchiste, qui donnera lieu à la présupposition rhétorique du lien entre capitalisme et stalinisme et au redéploiement de l'idée d'un capitalisme d'État).

\subsection{L'ethos du militant révolutionnaire contre l'ethos du prophète bourgeois}

21 L'affirmation identitaire du groupe Socialisme ou Barbarie (dont l'appellation, reprise à Rosa Luxembourg, affirme le parti pris militant hétérodoxe) se pose comme une évidence explicitée : la revue se définit à la fois comme un «organe de critique et d'orientation révolutionnaire" et comme un groupe de "camarades" au projet commun affirmé et défini : "les noyaux de l'organisation révolutionnaire, tel notre 
groupe, devront partir d'une base théorique ferme, diffuser la nouvelle conception des problèmes et la concrétiser toujours davantage " (1949: 4). Il est intéressant de confronter la mise en scène de soi du groupe à celles des autres revues intellectuelles. Tandis que Critique joue sur un ethos de modestie, annonçant, à la troisième personne et sans recours à des assertions en "nous ", qu'elle voudrait « donner un aperçu, le moins incomplet qu'il se pourra, des diverses activités de l'esprit humain dans les domaines de la création littéraire, des recherches philosophiques, des connaissances historiques, scientifiques, politiques et économiques» (Critique 1946 : 2), Les Temps Modernes met en œuvre un ethos beaucoup plus affirmé et péremptoire, utilisant le déictique « nous » et un ton assuré: "Nous ne voulons rien manquer de notre temps [...]. Serions-nous muets et cois comme des cailloux, notre passivité même serait une action. [...] L'écrivain est en situation dans son époque " (Sartre 1945: 4-5). S'exprimant "sans mollesse ", selon les propos d'Annie Cohen-Solal (1985: 338), en termes d'assurance «nous ne voulons", «nous nions que», «L'écrivain est en situation»- et d'explicitation - réfutation par tournures hypothétiques ${ }^{7}$-, le discours des Temps Modernes illustre, dans le ton et les formules qu'il adopte, une position d'énonciateur qui se veut inébranlable, ceci s'exprimant au travers d'un ethos surplombant, assertif et contraignant de prophète. Toutefois, si l'usage du «nous » est récurent, l'énonciateur n'est pas anonyme et affirme l'importance de l'individualité qui le signe: Jean-Paul Sartre, le directeur de la revue.

La proximité rhétorique de Socialisme ou Barbarie avec les assertions sartriennes en "nous" est évidente: "Nous pensons que ", "nous n'avons nullement peur $d[e]$ ", "Nous cherchons donc" (Socialisme ou Barbarie 1949 : 3-5). Reprenant dans une certaine mesure la rhétorique sartrienne, la revue de Castoriadis et Lefort en accentue le caractère homogène et assertif, déplaçant l'ethos du prophète vers celui du militant de groupe, suivant une mise en scène du locuteur en tant que voix groupale unifiée et anonyme, non en tant que représentant bourgeois d'un collectif d'intellectuels hétérogène et sériel. L'analyse de Daniel Ferrand peut dès lors être réorientée vers une dimension rhétorique, celui-ci évoquant la figure du «militant comme porte-parole » et précisant que «si le militant diffuse une théorie, ce n'est pas la sienne propre mais celle qui est élaborée par l'organisation; il en est le porte-parole » (Ferrand 2012 : 156). Ainsi, contre l'insistance récurrente des revues intellectuelles sur la liberté individuelle sous-tendant chaque article, qui n'engage que son auteur, l'ethos de militant révolutionnaire qui se construit dans Socialisme ou Barbarie engage au contraire la collectivité, le groupe de militants que Castoriadis souhaite véritablement transformer en organisation révolutionnaire dont l'intellectuel ne serait qu'un " porte-parole».

Une série de particularités discursives, en plus de l'absence de signature à la fin du texte, participent à la construction d'une voix énonciatrice collective et unifiée : l'existence et l'unité de groupe sont posées dès la première ligne comme une évidence $a$ priori; la cohésion et la cohérence idéologiques sont explicitées; le « nous » se veut être un nous exclusif et englobant, non une façade rhétorique; l'affirmation d'une appartenance de classe commune à la classe des «ouvriers manuels et intellectuels » rompt avec toute conception bourgeoise de l'engagement individuel; la théorie de l'action militante radicalise la dimension pratique et performative du discours; enfin la dénonciation de toute figure prophétique individuelle, qu'elle soit marxienne ou sartrienne, se fait au profit d'une collectivité de militants, de pairs, de «camarades » et 
se conçoit au travers de l'idéologie hétérodoxe d'une auto-organisation. Tous ces éléments se retrouvent assez exemplairement synthétisés dans l'extrait suivant :

Cette théorie révolutionnaire sur laquelle doit constamment s'appuyer l'action, quelle est-elle? Est-elle un dogme sorti armé de pied en cap de la tête de Marx ou d'un autre prophète moderne, et dont nous autres nous n'aurions comme mission que de maintenir sans tache la splendeur originelle ? [...] Nous avons déjà dit par là, que si nous nous considérons comme marxistes, nous ne pensons nullement qu'être marxiste signifie faire par rapport à Marx ce que les théologiens catholiques font par rapport aux Écritures (Socialisme ou Barbarie 1949 : 3-4).

On peut ainsi soutenir que l'anti-stalinisme et l'anti-dirigisme sans concession du texte de présentation, qui s'ouvre sur une critique ouverte de la bureaucratie en tant que problème fondamental de notre époque qui « aliène et mystifie » le prolétariat (ibid. : 1 et 4), se double d'une critique et d'un déplacement militant des positions idéologiques et rhétoriques des existentialistes. Précisons ici qu'une critique aussi vive de l'URSS sous l'ère stalinienne est, comme le suggère Sartre dans Les Racines de l'éthique, éminemment complexe et tend à apparaître comme un discours allant à l'encontre du développement du socialisme (Sartre $2015: 12$ ). Si cette critique est récurrente au sein $\mathrm{du}$ discours communiste orthodoxe à propos des marxismes hétérodoxes (principalement dans les années 1930 et 1940), il faut dans un même temps relever le pouvoir instituant du discours antisoviétique de Socialisme ou Barbarie qui rend dans le même temps possible l'intense critique se développant dans les années 1950 et permettant un dépassement de l'aliénation du monde intellectuel envers le modèle soviétique, dépassement perceptible dans les positions qu'occupent les revues Preuves, Arguments et Partisans dans les décennies postérieures.

\section{Une rhétorique de guerre froide : dialectique et troisième voie}

Dans la continuité de ces premières analyses sociodiscursives à partir de l'évolution des positions des revues, il est fondamental de s'attarder sur la traduction rhétorique d'un contexte politique singulier, celui de la progressive instauration d'une guerre froide influençant directement les positionnements au sein de la formation discursive marxiste. L'insistance constante de Socialisme ou Barbarie sur ce contexte, qui est par ailleurs qualifié de "Troisième Guerre Mondiale» (ibid.: 2), entraîne l'usage de nombreux déictiques spatio-temporels traduisant l'importance rhétorique d'une situation historico-sociale: "En nous présentant aujourd'hui », "La situation actuelle", "aussi bien en France que dans les autres pays", "dans la période actuelle», "les luttes actuelles ", "à une époque où ». À partir de ce constat, nous dégagerons deux éléments constitutifs d'une rhétorique marxiste de guerre froide, à savoir l'actualisation de la formule du "marxisme véritable» et la présupposition rhétorique du lien entre stalinisme et capitalisme. Socialisme ou Barbarie prépare en quelque sorte ce que Ory et Sirinelli nomment, par une formule intéressante, «la guerre froide des intellectuels » (2002: 155-188) et qui nous permet de considérer la période 1945-1949 comme un moment rhétorique et idéologique préparant le contexte intellectuel des années 1950. 


\subsection{La formule du « marxisme véritable »}

26

êrs éments discursifs peuvent être interprétés comm situation historique qui se cristallise tout particulièrement autour de formules relatives à une prétendue substance véritable du marxisme. Nous reprenons ici la notion de formule à Alice Krieg-Planque qui définit celle-ci comme un ensemble de formulations actualisées dans une formation discursive, investies d'une valeur idéologique et polémique et tendant vers un figement de la forme au profit d'une extinction du sens. Il sera dès lors question d'analyser les différentes actualisations de la formule «marxisme véritable » dans le texte de présentation de Socialisme ou Barbarie, par ailleurs présente dans plusieurs articles parus dans les revues d'immédiat après-guerre - relevons très sommairement l'utilisation de "marxisme véritable » par Alquié, dans "Marxisme ou cartésianisme " paru dans Les Temps Modernes, et de "véritable marxiste ", dans la présentation de La Nouvelle Critique qui se présente par ailleurs comme la revue $d u$ marxisme militant. Cette formule peut en outre s'actualiser selon diverses variantes telles que "véritable essence du marxisme ", "vérité du marxisme ", "nature du marxisme ", etc. Ces occurrences d'une même formule permettent de comprendre la position de la revue de Lefort et Castoriadis dans un contexte antagonique de guerre froide en ce qu'il induit certaines structures argumentatives témoignant d'une posture d'entre-deux, de double réfutation (du stalinisme et du capitalisme) et d'un besoin d'auto-affirmation.

Lexte de présentation mobilise donc un ensemble de formules gravitant autour de l'association sémique /marxisme/ + /vérité/. Relevons préalablement: "véritable essence du trotskisme ", la "nature du stalinisme", " nous pensons être les seuls à reprendre et à continuer l'analyse marxiste ", " Nous pensons que nous représentons la continuation vivante du marxisme» (Socialisme ou Barbarie 1949: 1-6). Ces formules traduisent la volonté et le besoin d'une autodéfinition univoque («l'analyse marxiste » et non « une analyse marxiste », « la continuation vivante du marxisme » et non « une continuation vivante d'un marxisme ») qui permet de clarifier une position de rejet tant de l'URSS, et partant des affirmations staliniennes, que du capitalisme, interdisant par là toute condamnation de réformisme : ce marxisme exclut les variantes.

Cette course au bon marxisme, à sa supposée substance véritable, révèle une escalade discursive de la part des intellectuels d'obédience marxiste donnant lieu à une rigidification, à une essentialisation, de cette idéologie. Si Socialisme ou Barbarie souhaite explicitement rejeter tout "dogme, sorti armé de pied en cap de la tête de Marx ", certaines formulations traduisent bien une attitude scientiste, à l'image de celle-ci : "nous pensons être les seuls à reprendre et à continuer l'analyse marxiste de l'économie moderne, à poser sur une base scientifique le problème du développement historique du mouvement ouvrier et de sa signification [...]» (Socialisme ou Barbarie 1949: 2, nous soulignons). Si ce scientisme parait proche du dogmatisme des communistes orthodoxes, il est principalement le résultat d'une volonté d'autolégitimation de la part d'un groupe minoritaire ancré au cœur d'un conflit idéologique où les pôles dominants (orthodoxie, exigences scientifiques, tendances néopositivistes) l'influencent et le contraignent.

Le caractère formulaire de l'idéologie marxiste amène dès lors les intellectuels de Socialisme ou Barbarie, plus ou moins malgré eux, à vider celle-ci de sa substance constitutive au profit d'un figement polémique des débats où l'un prétend détenir la 
vraie essence du marxisme tandis que l'autre la dégraderait. La violence des condamnations de la part des staliniens - on le voit très clairement dans les premiers articles de La Nouvelle Critique - envers ceux qu'ils nomment les réformistes ou «intellectuels désorientés » (La Nouvelle Critique 1948: 3), lorsqu'ils n'en font pas des prétendus agents du capitalisme, de même que l'opposition en retour des trotskistes et des hétérodoxes de tous bords, participent à la construction de formules figeant le discours à propos du marxisme dans sa dimension polémique et interdisant par là tout réel questionnement épistémologique de ces formules. Ainsi, La Nouvelle Critique fige à nouveau la substance du marxisme, sans jamais la délimiter positivement, en la résumant à une théorie sortie tout droit d'œuvres considérées comme des « doctrines»:

Il les gêne que le marxisme ne meure pas avec Marx, mais qu'on ne puisse aujourd'hui revendiquer le titre de marxiste sans se réclamer aussi de Lénine et de Staline, sans compter aussi avec l'immense apport théorique du léninisme et du stalinisme à la doctrine de Marx-Engels (ibid. : 10).

En réaction à ce discours marxiste-léniniste, Socialisme ou Barbarie suggère la possibilité de développer une conception, certes attentive à «maintenir et défendre les positions marxistes traditionnelles", mais avant tout soucieuse d'un renouvellement critique, d'un "nouvel examen" de cette tradition pouvant être "amendée», voire «remplacée » (1949:4). Cette dualité est la preuve d'une difficulté réelle de rompre totalement avec l'orthodoxie marxiste qui agit sur la formation discursive comme une position plus ou moins dominante, position que le groupe entend par ailleurs renverser. Les injonctions orthodoxes du supposé «marxisme véritable » constituent bel et bien un élément structurant de la formation discursive marxiste au sein de laquelle la position stalinienne et les exigences de scientificité influent sur les revues hétérodoxes en quête de légitimité politique et idéologique et au sein desquelles le recours doctrinaire à la tradition reste déterminant.

\subsection{Présupposition rhétorique du lien entre stalinisme et capitalisme}

31 En retour de cette logique d'influence de la position orthodoxe sur une production hétérodoxe, on peut analyser la présupposition rhétorico-idéologique entre stalinisme et capitalisme réalisée par Socialisme ou Barbarie comme un déplacement singulier de la formation discursive marxiste. Ainsi, dans une phrase telle que «Notre but sera de fournir des outils de travail aux ouvriers avancés, à une époque où la complexité des problèmes, la confusion qui règne partout et l'effort constant des capitalistes et surtout des staliniens pour la mystification de tous à propos de tout nécessitent un effort sans précédent dans cette direction» (ibid.: 6, nous soulignons), le complément du nom «des capitalistes et surtout des staliniens pour la mystification de tous à propos de tout " établit une analogie entre mystifications capitaliste et soviétique, et participe dans le même temps, par sa nature grammaticale, à la présupposition rhétorique de cette analogie. En effet, en plaçant le rapport entre capitalisme et stalinisme dans un complément du syntagme "effort constant", le locuteur considère comme un présupposé ce rapport ainsi que l'effectivité de la mystification propre aux deux régimes politiques, leur analogie ne pouvant être contestée puisque présupposée grammaticalement. 
32 En n'étant pas formulée comme une idée développée, explicitée et assumée par le locuteur mais en étant présentée comme une évidence a priori, cette tournure argumentative contraint l'interlocuteur, le coénonciateur, à admettre ce présupposé rhétorique. En effet, celui-ci est construit par le locuteur Socialisme ou Barbarie comme un discours entendu sans pour autant relever d'une doxa avérée. Véritable stratégie énonciative, le fait de présenter comme évident, et sous l'apparence d'une objectivité ("à une époque où »), ce rapport entre capitalisme et stalinisme participe d'un effet point de vue amenant à une absence de remise en cause de la présupposition rhétorique considérée par le locuteur comme admise et partagée. Le texte passe dès lors sous silence, tout en l'établissant sous l'apparence d'une évidence, l'analogie pouvant être formulée comme suit : « les capitalistes et les staliniens mettent en œuvre un effort constant de mystification de tous et de tout, et cet effort est commun ». Cette stratégie argumentative, retorse par sa dimension implicite et par la contrainte interprétative qu'elle induit, fonctionne comme une hétérogénéité énonciative constitutive, non montrée, telle que l'entendent François Provenzano et Émilie Goin à la suite de Jacqueline Authier-Revuz :

En simplifiant quelque peu, on pourrait dire que dans le cas d'une hétérogénéité montrée, l'e2 sera particularisé et pointé comme une autre personne ou personnage dont le L1/E1 rapporte les propos, tandis que dans le cas de l'hétérogénéité constitutive, l'e2 correspondra plutôt à une «formation discursive " qui n'est pas rattachée à une instance particulière pointée comme "autre ", mais plutôt à un ensemble plus ou moins abstrait de croyances marquées socialement et idéologiquement (Goin et Provenzano 2015 : 77-78).

33 Ainsi le L1/E1 Socialisme ou Barbarie met en scène une doxa construite (12/e2) qui sert comme formation discursive sous-tendant et soutenant le point de vue de ce L1/E1, insinuant par là un ensemble de «croyances marquées socialement et idéologiquement ». Cependant, le L1/E1 construit véritablement cette doxa sur base d'une idéologie marxiste hétérodoxe sans que celle-ci fonctionne effectivement comme telle dans les croyances collectives. Ce tour de force rhétorique, qui construit une apparence de dialogisme et une instance énonciative tierce, amène les interlocuteurs à intégrer une présupposition hautement idéologique sous couvert d'une évidence partagée et à déplacer la formation discursive en y instituant des idéologèmes précis.

Il est en outre utile de noter, à l'appui de cette analyse, que le texte de présentation adresse le qualificatif de «bâtards intermédiaires » (Socialisme ou Barbarie 1949: 3) aux variantes capitalistes, ceci pouvant être interprété comme une nouvelle attaque allusive envers l'URSS analysée par le groupe comme un dérivé du mode de production capitaliste, comme une "exploitation et une oppression pires que sous le capitalisme bourgeois » (Blanchard 2007 : 8). Cette interprétation du modèle économique et social de l'URSS amène dès lors à un déplacement de la formation discursive marxiste vers une hétérodoxie assumée, qui ne conçoit pas ce régime comme un état communiste, comme le suggère le dogmatisme orthodoxe, ni comme un état ouvrier dégénéré, comme l'analyse la mouvance trotskiste, mais comme un véritable capitalisme d'État ${ }^{8}$. Cette orientation discursive et idéologique présupposée par le groupe Socialisme ou Barbarie influe dès lors sur l'institution discursive et idéologique du marxisme et agit comme une réaction à la détermination de l'orthodoxie contenue dans la formule « marxisme véritable ». 


\section{La praxis de groupe contre la logique pratico-inerte de la formation discursive}

Ces dernières remarques devront être comprises à partir d'un commentaire théorique autour des concepts sartriens de praxis et d'anti-praxis structurant les analyses que nous avons menées à propos des rapports entre les textes de présentation des revues et la matérialité de la formation discursive marxiste. Afin de comprendre la logique dialectique à l'œuvre entre le discours d'intervention de Socialisme ou Barbarie et cette formation discursive, il sera nécessaire d'analyser leurs rapports au travers du dépassement de la dialectique entre praxis et anti-praxis, par la mobilisation des différentes observations socio-rhétoriques que nous avons réalisées, en concevant la formation discursive comme un ensemble pratico-inerte synthétisant le double fonctionnement praxis/anti-praxis (Sartre $1960: 296-329$ ).

Les exigences d'engagement des revues intellectuelles de l'immédiat après-guerre, radicalisées par le militantisme de groupe de Socialisme ou Barbarie, peuvent se lire comme un projet d'action et de transformation de la matérialité sociale, des structures politiques et idéologiques instituées, d'un état donné des rapports de production et de domination au sein d'une formation discursive (par exemple la domination de l'orthodoxie du marxisme véritable contre la position dominée de l'idée hétérodoxe d'un capitalisme d'État). L'analyse du processus dialectique entre praxis et anti-praxis considère l'action humaine comme le travail de transformation (praxis) de la matière en une matière ouvrée'. Dans le même temps, celle-ci agit sur la pratique humaine suivant un mouvement dialectique en retour induit par l'action de la matière elle-même (antipraxis), qui réifie et rend inerte la praxis de l'homme, qui fige sa pratique discursive dans une rhétorique instituée.

Ainsi, la praxis discursive de Socialisme ou Barbarie peut, dans un premier temps, se comprendre comme un projet de transformation de la matérialité sociodiscursive. Insistant, en bonne marxiste, sur la corrélation entre théorie et pratique révolutionnaires, sur le rapport entre la connaissance théorique et l'activité transformatrice qui en découle, la revue de Lefort et Castoriadis théorise et assume ce rôle d'action-transformation, cette performativité de l'acte théorico-pratique. La revue intellectuelle agirait, selon ce premier mouvement, sur la matérialité discursive en tant qu'organe collectif faisant partie intégrante de celle-ci et transformant par là-même son agencement, tel un discours d'intervention. On voit en quoi l'action de Socialisme ou Barbarie influe sur la matérialité discursive de la formation discursive marxiste, déplaçant celle-ci vers un anti-stalinisme dur et sans concession et vers une critique du trotskisme, ceci amenant à la formulation d'une idée selon laquelle le pouvoir politique de l'URSS s'apparente davantage à un capitalisme d'État qu'à un état ouvrier dégénéré. De même, tout en reprenant l'ethos de prophète des Temps Modernes, elle le déplace vers une dimension militante-intellectualiste assumée. La revue rend dès lors possible une pensée hétérodoxe singulière par sa formulation, elle permet l'émergence, du moins le déplacement, le mouvement instituant, d'une idée, celle du capitalisme d'État, au sein d'une formation discursive.

En retour de ce premier mouvement praxique, la revue, en étant elle-même un objet collectif travaillé par une matérialité discursive déjà instituée (les structures rhétoriques et idéologiques d'un état donné de la formation discursive), est en même temps soumise au pouvoir toujours contraignant de la matière qui la domine et la 
détermine. Conçue dans un premier temps comme une activité humaine d'invention et d'intervention pratique, la revue subit en retour l'action de l'anti-praxis, résultat dialectique de la praxis, produite par la matière discursive qu'elle a travaillée (et qui la travaillait déjà). La formation discursive sur laquelle agissent les différentes revues produit dans le même temps une action praxique qui fige celles-ci et les contraint à un empêtrement inerte dans la matérialité sociale et discursive. Les structures rhétoricoargumentatives reproduites dans les revues que nous avons étudiées (l'ancrage dans un registre polémique, la reproduction de l'orthodoxie contenue dans la formule du marxisme véritable, le topos de la libre discussion contrainte) illustrent ce pouvoir d' anti-praxis de la formation discursive sur les discours d'intervention.

Cette mise en lumière d'une logique dialectique propre à l'ancrage d'un discours dans une formation discursive nous amène à considérer celle-ci comme un ensemble praticoinerte tel que l'entend Sartre, c'est-à-dire comme une structure matérielle contraignant par son pouvoir d'inertie les actions humaines qui ne peuvent agir que par et au travers d'elle. Ces exigences d'inertie de la matérialité délimitant la formation discursive enjoignent tout discours à agir suivant une pratique empêtrée dans l'inertie matérielle : «Seule la matière elle-même, cognant sur la matière, pourra les [les projets des hommes] désagréger. Le sens du travail humain, c'est que l'homme se réduit à la matérialité inorganique pour agir matériellement sur la matière et changer sa vie matérielle " (ibid. : 288-289). Il faut ici évoquer l'importance des logiques de pouvoir et de domination inhérentes à la structuration même de la formation discursive qui contraignent plus ou moins l'action humaine en fonction de l'accentuation de ces logiques. Ainsi, dans le cas d'une formation discursive hautement idéologique telle que celle du marxisme, les productions singulières se voient contraintes par un pouvoir propre à une institution plus ou moins rigide de la matière et à des positions dominantes reproduisant le pouvoir d'inertie de celle-ci (c'est le cas de l'orthodoxie marxiste-léniniste qui agit comme position dominante). Comme le note Sartre, les significations sont des forces qui s'opposent (ibid.: 289) et qui s'affrontent plus ou moins durement à une époque, dans une spatialité et au sein d'une organisation sociale données. Il apparaît dès lors nécessaire de comprendre dans le même temps la singularité discursive de la revue intellectuelle au travers de son pouvoir de transformation de la matière sous l'action de sa praxis de groupe, c'est-à-dire « en tant qu'il [le groupe] lutte en lui-même contre l'inertie pratique qui l'affecte » (ibid. : 453), qu'il réunit des forces organisées s'opposant à la domination et au pouvoir d'inertie de la matérialité d'une formation discursive.

Pour finir, nous formulerons, au départ de ces remarques, plusieurs relances sous forme de questionnements théoriques : comment dépasser le pouvoir pratico-inerte de la matière discursive? Peut-on penser les logiques d'auto-organisation, d'autoappropriation et d'autogestion du savoir par les revues, leurs pratiques éditoriales collectives, leurs praxis discursives conscientes de l'inertie sociodiscursive et, partant, leur exigence constante d'une innovation rhétorique et idéologique concertée, comme autant d'éléments luttant avec la matérialité inerte et réifiante ? La définition de la revue comme création consciente, critique et dialoguée d'un groupe agissant sur tous les niveaux de la matière (discursive, éditoriale, historique, sociologique), c'est-à-dire comme discours d'intervention groupale telle que nous avons pu l'analyser au travers de la radicalité de Socialisme ou Barbarie, rend-elle possible un renversement de la domination de la matière sur les activités humaines, celles-ci n'étant dès lors plus conçues de manière sérielle mais comme le fruit d'une conscience d'un groupe unifié et 
organisé ? Bien que Sartre relève que "l'invention, avant même d'être faite, peut être, dans certaines circonstances de la production, une exigence de l'Être pratico-inerte " (ibid.: 301-302), ne pourrait-on pas considérer d'autres «circonstances de la production " - à l'instar de l'autogestion du savoir et de la matière discursive rendue possible par la particularité des revues intellectuelles agissant telles des groupes organisés - comme permettant un dépassement, du moins un déplacement, des rapports de domination entre la matérialité discursive et l'action humaine créatrice, dès lors définie comme une praxis de groupe?

\section{BIBLIOGRAPHIE}

\section{Sources primaires}

Alquié, Ferdinand. 1946. « Marxisme ou cartésianisme? », Les Temps Modernes 8

Aron, Raymond. 1946. « Une constitution provisoire », Les Temps Modernes 9

Caillois, Roland. 1946. « La pensée politique de Raymond Aron », Critique 5

Collectif. 1946. «CRITIQUE », Critique. Revue générale des publications françaises et étrangères 1

Collectif. 1948. « Au lecteur », La Table Ronde 1

Collectif. 1948. « Présentation », La Nouvelle Critique. Revue du marxisme militant 1

Collectif. 1949. « Socialisme ou Barbarie », Socialisme ou Barbarie. Organe de critique et d'orientation révolutionnaire 1

Lefort, Claude. 1946a. « Double et triple jeu. Réponse à M. Merleau-Ponty et à P. Hervé », La Jeune Révolution 2

Lefort, Claude. 1946b. « La déformation de la psychologie, du marxisme et du matérialisme ou les essais de M. Naville ", Les Temps Modernes 13

Sartre, Jean-Paul. 1945. «Présentation », Les Temps Modernes 1

Weil, Éric. 1946. «À propos du matérialisme dialectique », Critique 1

\section{Sources secondaires}

Angenot, Marc. 2008. Dialogue de sourds. Traité de rhétorique antilogique (Paris : Mille et une nuits)

Angermuller, Johannes. 2013a. Le champ de la théorie. Essor et déclin du structuralisme en France

(Paris : Hermann)

Angermuller, Johannes. 2013b. Analyse du discours poststructuraliste : les voix du sujet dans le langage chez Lacan, Althusser, Foucault, Derrida, Sollers (Limoges : Lambert-Lucas)

Authier-Revuz, Jacqueline. 1984. « Hétérogénéité(s) énonciative(s) », Danon-Boileau, Laurent (dir.), Langages 73

Blanchard, Daniel. 2007. «Préface », Socialisme ou Barbarie. Anthologie (La Bussière : Acratie)

Boschetti, Anna. 1985. Sartre et « Les Temps Modernes » (Paris : Minuit) 
Cohen-Solal, Annie. 1985. Sartre. 1905-1980 (Paris : Gallimard)

Curatolo, Bruno et Jacques Poirier, dir. 2002. Les Revues littéraires au XXe siècle (Dijon : Éditions Universitaires de Dijon)

Ferrand, Daniel. 2012. « Militer », Klimis, Sophie, Philippe Caumières \& Laurent Van Eynde (dir.). Socialisme ou Barbarie aujourd'hui. Analyses et témoignages (Bruxelles : Facultés Universitaires Saint-Louis)

Goin, Emilie \& François Provenzano. « Le genre comme médiation énonciative. L'exemple de l'effet de politisation dans le roman témoignage et la harangue ", Ablali, Driss, Sémir Badir \& Dominique Ducard, dir. 2015. En tous genres. Normes, textes, médiations (Louvain-la-Neuve : Academia-L'Harmattan)

Gottraux, Philippe. 1997. « Socialisme ou Barbarie ». Un engagement politique et intellectuel dans la France de l'après-guerre (Lausanne : Payot Lausanne)

Krieg-Planque, Alice. 2009. La notion de "formule » en analyse du discours. Cadre théorique et méthodologique (Besançon : P. U. Franche-Comté)

Maingueneau, Dominique. 2011. «Pertinence de la notion de formation discursive en analyse du discours ", Langage et Société 135 (Paris : Éditions de la Maison des sciences de l'homme)

Matonti, Frédérique. 2004. Intellectuels communistes. Essai sur l'obéissance politique (Paris : La Découverte)

Ory, Pascal et Jean-François Sirinelli. 2002. Les Intellectuels en France. De l'affaire Dreyfus à nos jours (Paris : Colin)

Pêcheux, Michel. 1975. Les Vérités de la Palice. Linguistique, sémantique, philosophie (Paris : Maspero) Pêcheux, Michel. 1990. "Analyse de discours. Trois époques ", Maldidier, Denise éd. L'Inquiétude du discours. Textes de Michel Pêcheux (Paris : Éditions des Cendres)

Peyre, Yves. 2002. «Généalogie de la revue moderne », Curatolo, Bruno et Jacques Poirier (dirs), Les Revues littéraires au XXe siècle (Dijon : Éditions Universitaires de Dijon)

Provenzano, François. 2017. « De l'inventio à l'intervention : perspectives rhétoriques sur l'invention » (non publié)

Sartre, Jean-Paul. 1960. Critique de la raison dialectique. Théorie des ensembles pratiques (Paris : Gallimard)

Sartre, Jean-Paul. 2015. Les Racines de l'éthique, Bourgault, Jean \& Grégory Cormann (dirs), Études sartriennes 19 (Bruxelles : Ousia)

Veyne, Paul. 2014. Et dans l'éternité je ne m'ennuierai pas. Souvenirs (Paris : Albin Michel)

\section{NOTES}

1. Voir, pour une description de ces contextes, Ory et Sirinelli $2002: 143-188$.

2. Voir à ce propos les réflexions de François Provenzano sur le rapport entre inventio et intervention (Provenzano 2017).

3. Normalien intégré par Merleau-Ponty dans Les Temps Modernes dès 1945, Lefort termine dixième à l'agrégation de philosophie en 1949. Castoriadis, diplômé de l'Université d'Athènes et de l'Institut français d'Athènes, intègre l'OCDE en tant qu'économiste en 1948. Il semble évident que, en la comparant au capital de La Nouvelle Critique, la revue militante Socialisme ou Barbarie 
dispose d'une légitimité bien plus grande, également acquise par sa proximité avec Les Temps Modernes dans laquelle Boschetti voit l'exemple même de l'accumulation de figures au capital symbolique très élevé.

4. Malgré l'usage de ces pseudonymes, il est évident que les milieux intellectuels militants de l'après-guerre savaient qui composait ce groupe "Socialisme ou Barbarie». Toutefois, le pseudonyme soulève un ensemble de réels problèmes dans l'analyse d'un ethos discursif et, plus généralement, dans la théorie de l'analyse du discours.

5. Psychanalyste ayant étudié à l'ENS et à Harvard avant la fin des années 1940, il obtient l'agrégation de philosophie en 1950 et devient maître-assistant à la Sorbonne.

6. Cet usage de l'îlot textuel (terme d'Authier-Revuz) est extrêmement récurrent dans les revues de l'après-guerre : à propos des « socialistes » dans La Nouvelle Critique, des écrivains " engagés » dans La Table Ronde, de la « nature » humaine dans Les Temps Modernes.

7. Tournure que l'on retrouve également chez Lefort et Merleau-Ponty, notamment dans « La querelle de l'existentialisme ».

8. Si plusieurs intellectuels anarchistes et trotskistes avaient déjà analysé, au cours des $19^{\mathrm{e}}$ et $20^{\mathrm{e}}$ siècles, les dérives autoritaires et le fonctionnement industriel de l'URSS comme un capitalisme d'État, rares étaient ceux qui intégraient ce constat à une analyse marxiste hétérodoxe, c'est-àdire se basant sur une critique de l'économie politique de l'URSS à partir des logiques de lutte de classe, de lutte idéologique, des analyses de l'aliénation par la propriété collective, de la main d'œuvre soviétique comme élément de la plus-value de la marchandise industrielle et de son autoritarisme hiérarchique et dirigiste.

9. Sartre entend par ce terme le résultat du travail humain sous l'action de sa praxis.

\section{RÉSUMÉS}

L'analyse sociodiscursive des textes de présentation des revues intellectuelles de l'immédiat après-guerre, et plus particulièrement de celui de Socialisme ou Barbarie, met en lumière une série d'éléments rhétoriques et argumentatifs propres au contexte socio-historique dans lequel elles évoluent, aux logiques éditoriales structurant l'espace des revues et à la formation discursive marxiste. D'une part, l'ancrage de ces productions intellectuelles dans un registre polémique de dispute idéologique, la reproduction de la formule figée du "marxisme véritable " ainsi que l'usage d'îlots textuels ironiques (éléments reprenant les paroles de l'adversaire pour le discréditer) constituent autant d'exemples de la reproduction d'un état institué du discours intellectuel, d'une utilisation par les revues d'un matériau discursif préconstruit. D'autre part, le déplacement que Socialisme ou Barbarie opère d'un ethos de prophète vers un ethos de militant de groupe, de même que la présupposition rhétorique du lien entre stalinisme et capitalisme d'État constituent des singularisations, des déterritorialisations, de ce discours préconstruit, que les revues tendent à déplacer et à transformer. Cette logique dialectique entre une reproduction de l'institution discursive et un pouvoir de singularisation instituante doit être comprise et nuancée à partir d'une réflexion philosophique matérialiste. Dans le cadre de cette conception, le mouvement dialectique à l'œuvre entre une création discursive - dans ce cas l'intervention militante de Socialisme ou Barbarie - et un état institué du discours - dans ce cas le dogmatisme marxiste - doit être pensé grâce à une réflexion théorique relative à la notion de praxis de groupe, qui permet de dépasser ce mouvement entre une production discursive individuelle et 
une communauté discursive au profit d'une mise en avant des particularités rhétoriques et du projet critique de la revue intellectuelle.

The sociodiscursive analysis of post-war intellectual reviews' editorials, especially Socialisme ou Barbarie, gives rise to rhetorical and argumentative interpretations determined by the sociohistorical context, by reviews' editorial features and by the Marxist discursive formation. On the one hand the polemic dimension of these discourses (structured by a logic of ideological dispute), the reproduction of the fixed formula "marxisme véritable" and the use of ironical "îlots textuels" (parts of discourse that quote words of the opponent to discredit him) are all rhetorical examples of the influence of an intellectual discourse's instituted state. On the other hand the militant ethos developed by Socialisme ou Barbarie and the presupposition of the link between Stalinism and State capitalism are parts of a discursive innovation. This dialectical logic between a discursive determination and a power of innovation has to be studied from a materialist point of view. Thus the dialectical movement between a discursive creation - for example the militant activism of Socialisme ou Barbarie - and a discourse's instituted state - for example the Marxist dogmatism - must be understood on the basis of different materialist notions. Also the notion of "group praxis" allows to overpass this movement between determination and innovation. Consequently the possibility arises to think the rhetorical particularities and the critical process of intellectual reviews.

\section{INDEX}

Mots-clés : formation discursive, matérialité, marxisme, praxis de groupe, Socialisme ou Barbarie

Keywords : discursive formation, group praxis, marxism, materiality, Socialisme ou Barbarie

\section{AUTEUR}

\section{THOMAS FRANCK}

Université de Liège, Groupe COnTEXTES et Groupe de Recherches Matérialistes 